\title{
Efficient Re-Ranking of Images from the Web using Bag based Method
}

\author{
S.Keerthana \\ PG Department of Computer \\ Science \\ sona college of technology \\ Salem-05, TN, India
}

\author{
R.C.Narayanan \\ PG Department of Computer \\ Science \\ sona college of technology \\ Salem-05, TN, India
}

\author{
K.Krishnamoorthy \\ PG Department of Computer \\ Science \\ sona college of technology \\ Salem-05, TN, India
}

\begin{abstract}
1. ABSTRACT
An image retrieval system is a computer system for browsing, searching and retrieving images from a large database of digital images. Given a textual query in traditional text based image retrieval (TBIR),relevant images are to be re ranked using visual features after the initial text based image search. In this paper, we propose a new bag based re ranking framework for large scale TBIR. We compute this problem as Multiple Instance Learning and Generalized Multiple Instance (GMI) learning method. To address the ambiguities on the instance labels in the positive and negative bags we propose a GMI settings. Also the user log performs the operation of individual user interaction with the system which improves the performance of image retrieval.
\end{abstract}

\section{General Terms}

Multiple Instance, Generalized Multiple Instance, Image

Re- ranking, Text Based Image Retrieval

\section{Keywords}

Image retrieval, Re-ranking, Search engine

\section{INTRODUCTION}

The image ranking as an effective way to improve the results of Web based image search has been adopted by current commercial search engines. Given a query keyword a pool of images are re-ranked by the search engines based on the query. By asking the user to select a particular image from the pool, the remaining images are re-ranked based on the user selected image. To avoid the ambiguities in the re-ranked process and to achieve an effective and efficient re-ranking process we introduce a Bag Based Re-ranking approach. It performs

- Automatic annotation process by K-means algorithm which split the positive and negative bag that contains relevant and irrelevant images respectively.

- GMI-SVM process which perform bag based reranking effectively.

- $\quad$ Perform user log operation for individual user log in.

For this, first we partition the relevant images into clusters by using visual and textual features .here we use MI learning method, we treat each cluster as a bag and the images inside the cluster as instances.
In the GMI learning process, Automatic bag annotation process can be used to find positive and negative bags for training classifiers. User log can separate log for each user so that when the same user gives the same query again, directly the re-ranked clusters can be displayed to them from the cache memory maintained, in order to avoid user interaction for the ordering of the representative images. It also saves the execution time of the query.

\section{RELATED WORK}

Web-scale image search engines mostly use keywords as queries and rely on surrounding text to search images. It is well known that they suffer from the ambiguity of query keywords [1]. For example, using "apple" as query, the retrieved images belong to different categories, such as "red apple", "apple logo", and "apple laptop". Online image reranking has been shown to be an effective way to improve the image search results [2]. Partition the relevant images into clusters by using visual and textual features. MI learning method explains this problem [3].automatic bag annotation method perform much better re ranking than existing image re ranking method [4],[5]. A real-time textual query based personal photo retrieval system by leveraging millions of web images and their associated rich textual descriptions. After a user provides a textual query (e.g., "water"), our system exploits the inverted file to automatically find the positive web images that are related to the textual query "water" as well as the negative web images that are irrelevant to the textual query. Based on these methods it will automatically retrieve relevant and irrelevant web images [6]. Database containing a large number of images and with high precision is still an arduous manual task. To overcome the download restriction, it will use web search instead of an image search. This search can generate thousands of images. Since the objective in this work is to harvest a large number of images of a particular class automatically, and to achieve this with high precision [7]. RF increases the retrieval performance to the fact that it enables the system to learn what is relevant or irrelevant to the user across successive retrieval-feedback cycles [8]. Bag annotation method can achieve better retrieval performance compared to [4], [5]. This process is similar to [9]. To facilitate annotation process the bag ranking score can be used [10]. One leading framework for image object mining is the bag of words approach. the main motive is to encode an image as a collection of visual words of the quantized features. Here they use pseudo positive images produced in response to the original query. It gradually refines the query language model. Most of the work have emphasized on extracting information from data stored in database [11]. 


\section{PROPOSED WORK}

After the user log in, initially user log displays the information about the previous user recently searched images. From that a particular query selected by the user or a new query given by the user which retrieves images from the database. In the existing system, classification of images can be displayed by means of semantic signature. In our approach visual and textual features can be compared with the user selected image by means of shape, color and texture. In the annotation bag process K-means algorithm can be used to split the positive and negative bags which contain relevant and irrelevant images respectively.

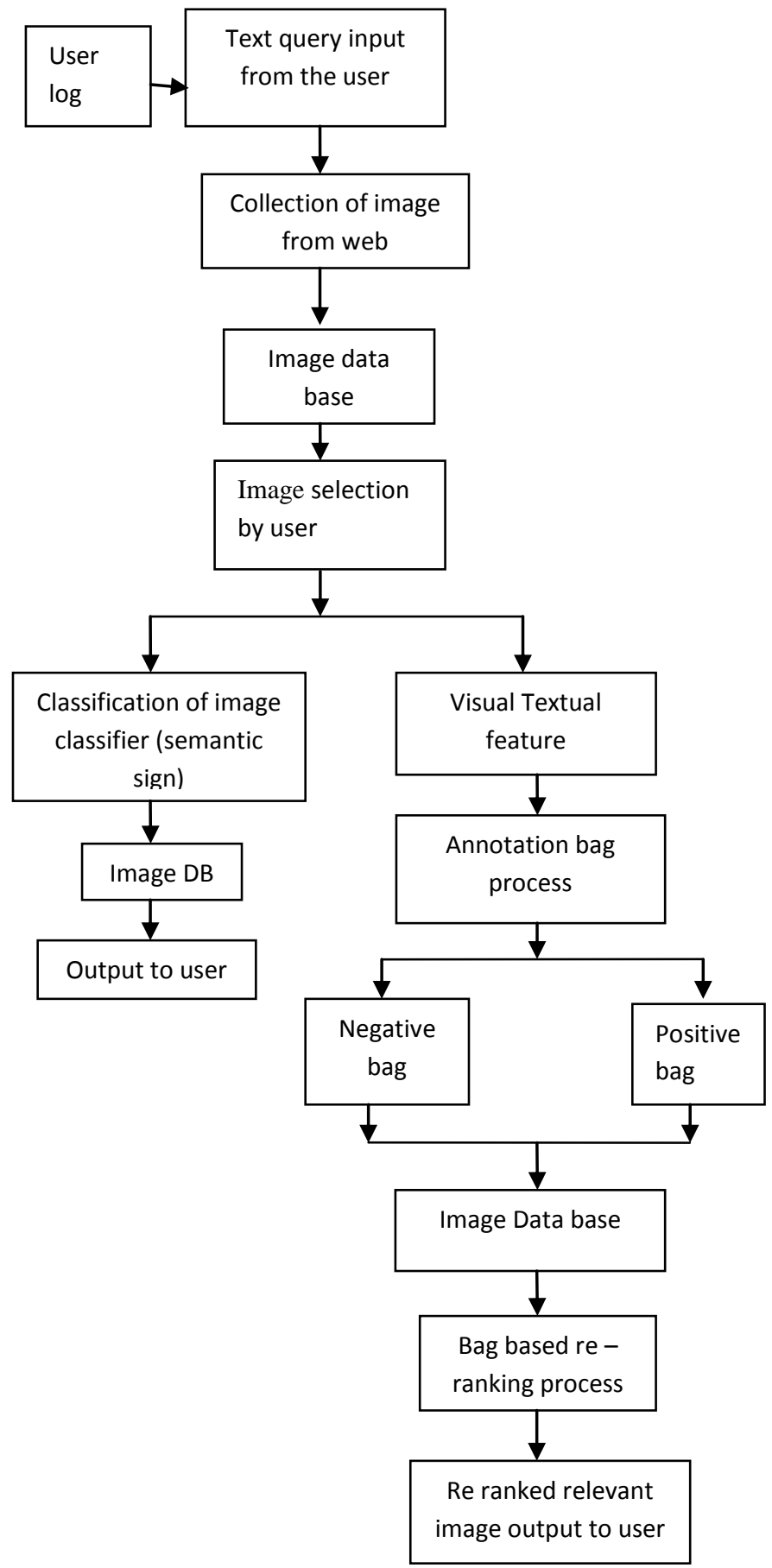

Fig 1 System architecture
Here positive bag only consider for re-ranking operation, this can send to the bag based re-ranking process which perform GMI learning method. This will provide an effective and efficient re-ranked image output to the user. Generally it performs the following five operations.

1. Construction of web image repository

2. Discovery of reference classes

3. Bag annotation process

4. Image Re-Ranking

5. Performance comparison

\subsection{Construction of web image repository}

Initially user log is performed which provide an authentication to each user. Depends on the user given query, collection of the images can be stored in the database

\subsection{Discovery of Reference classes}

Here the user given query relevant words are searched and it provides an easy access for relevant images. It will contain maximum of three relevant words which is relevant to the query word. This process contains two operations such as keyword expansion and selects reference classes respectively. In the keyword expansion, the relevant previous word and next word images queries are listed and which is already stored in the database that could be searched by the corresponding users. In the select reference classes corresponding images could be displayed to the user.

For a keyword q, we automatically define its reference classes through finding a set of keyword expansions E(q) most relevant to $q$, to achieve this a set of images $S(q)$ are retrieved by the search engine using $q$ as query based on textual information. keyword expansions are found from the words extracted from the images in $S(q)^{3}$.

\subsection{Bag Annotation Process}

In bag annotation process, visual and textual features such as color, shape and textures are considered and compared with user selected image. Here each image color could be counted and that can be counted and stored in database. These counting can be compared with remaining other images. For this we can use instance ranking score. This can calculate positive and negative bag images. $\mathrm{S}\left(\mathrm{B}_{\mathrm{I}}\right)$ perform this task .. Depends on the ranking percentage the chart could be plotted which represent relevant and irrelevant images percentage. By using this user can find relevant image percentage score. The image keyword relationship compares the total image versus image keyword shows in fig 4.

\subsection{Image Re-Ranking}

In this process input is selected by the user and the corresponding output can be displayed by Re Ranking of images. Here the positive bag and negative bag percentage could be calculated by means of Generalized Multiple Instance(GMI) Learning process. If the output ratio exceeds 69 percent then it moves to positive bag otherwise it moves to negative bag. This negative bag images are irrelevant that cannot be considered here. In fig.5, it shows the re-ranking 
percentage for every image. Depends on the number of relevant images the percentage could be calculated for every image in the database.

\subsection{Performance Comparison}

The proposed system is expected to deliver the below performance in comparison with the existing system and the same is interpreted below.

\begin{tabular}{|l|l|l|}
\hline Keyword & $\begin{array}{l}\text { Image re ranking } \\
\text { before process }\end{array}$ & $\begin{array}{l}\text { Image re ranking } \\
\text { after process }\end{array}$ \\
\hline Apple & $10 \%$ & $20 \%$ \\
\hline Fox & $15 \%$ & $37 \%$ \\
\hline Tajmahal & $12 \%$ & $15 \%$ \\
\hline
\end{tabular}

Table 1. Approximate Performance Measure

This system provide an more efficient and security compare to the existing method. The keyword relationship performance can be viewed in fig. 5 and the relevant images ranking score performance can be shown in fig.7.

\section{MATHEMATICAL MODEL}

\subsection{Construction of Image Database with Text}

Initially we are going to collect all type of images on the Internet (Eg: Google). This can collect all kind of images with text to a large collection of image data base.

\subsubsection{Initial Ranking}

For each retrieved relevant image $\mathbf{x}$, an instance ranking score can be defined as follows:

$$
r(X)=-\tau+\frac{1}{\delta}
$$

Where $\delta$ is the total number of tags in image $\mathrm{x}$ and $\tau$ is the rank Position of the query tag $\mathrm{q}$ in the tag list of an image $\mathrm{x}$. $\tau_{i}<\tau_{j}$ and $i \neq \mathrm{j}$ then we have $r(X i)>r(X j)$. In other words, when one relevant image contains the textual query $\mathrm{q}$ at the top position in its tag list, this image will be assigned a higher ranking score. When the positions of the query tag $\mathrm{q}$ at the top position in its tag list, this image will be assigned a higher ranking score. When the positions of the query tag $\mathrm{q}$ are the same for the two images $\tau_{i}=\tau_{j}$. the ranking score is decided by $\delta i$ and $\delta j$.

\subsection{Applying Bag Annotation for Re - ranking the Database Images}

After the user provides a textual query $q$ (e.g., "fox"), our system exploits the inverted file method to automatically find relevant web images whose surrounding text contains the textual query tag $q$, as well as irrelevant web images whose surrounding text do not contain $q$.

Each image is considered as an "instance". To construct "bags", we partition the relevant images into clusters using the k-means clustering method based on visual features and textual features. After that, each cluster is considered as a bag.

\subsubsection{Bag Annotation Process}

In bag annotation process, for each bag $\mathrm{B}_{\mathrm{I}}$, its bag ranking score $\mathrm{S}\left(\mathrm{B}_{\mathrm{I}}\right)$ is defined as the average instance ranking score,

$$
S(\mathrm{BI})=\frac{\sum \mathrm{x} \in \mathrm{BI} \mathrm{r}(\mathrm{x})}{|\mathrm{x}|}
$$

Where $\left|\mathrm{B}_{\mathrm{I}}\right|$ stands for the cardinality of a bag $\mathrm{B}_{\mathrm{I} \text {. }}$

\subsection{Implementing the Generalized Multiple- Instance (GMI)}

It performs the bag based re ranking process. To facilitate GMI learning methods in our framework, we have to annotate positive and negative bags to train classifiers. In our automatic bag annotation method, the top ranked bags with higher bag ranking scores are used as pseudo positive bags and the same number of pseudo negative bags is obtained by randomly sampling a few irrelevant images.

\subsubsection{GMI Learning Method}

The instance of positive and negative bag,

$\sum_{\mathrm{i}: x \in B i} \frac{\mathrm{y}+1}{2} \geq \mu|\mathrm{Bi}|$ for $\mathrm{Y}_{\mathrm{i}}=1$, and

$\sum_{\mathrm{i}: x \in B i} \frac{y+1}{2} \leq \gamma|\mathrm{Bi}|$ for $Y_{I}=-1$.

Where,

$$
\begin{aligned}
& \mu \rightarrow \text { positive bag } \\
& \gamma \rightarrow \text { negative bag }
\end{aligned}
$$

\section{4 exploiting user logs for user log in}

An effective exploitation of user logs for improving the performance of image retrieval system is to reduce the number of user interactions required before satisfactory results are achieved. The system assumes the availability of user logs from previous user interactions for a given query concept. Thus the system is to exploit the existing user logs to improve the performance of the image retrieval system.

For user log, membership function can be used for every user logging in. The classification algorithm produces the list of image ids from metadata repository.

\section{RESULTS}

Here the input is given by the user from the collection of images in fig.2. Depends on the user given feedback image the corresponding relevant images result will be produced in the fig 3 . The bag annotation process can perform an effective Re-ranking operation. Fig 3 shows the source and corresponding matching image that produces percentage of matching and their bag status of the input image. This gives an effective rank of that particular input image. By using this user can find the percentage of the input images and their bag 


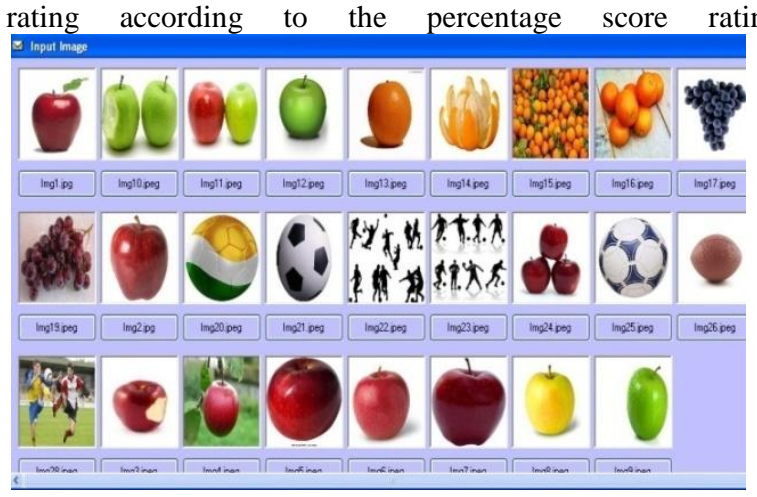

Fig 2.input image

\begin{tabular}{|c|c|c|c|c|}
\hline Process & & & & \\
\hline \multicolumn{5}{|c|}{ Q Records. } \\
\hline Rank & SourceImar & - MatchImare & Percentage & Bax \\
\hline 9 & Im833.jpeg & Ing12.jpeg & 2 & Negative ... \\
\hline 41 & Ime33.jpeg & Img35.ipeg & 2 & Negative ... \\
\hline 57 & Imp33.jpeg & Img18.ipeg & 2 & Negative ... \\
\hline 58 & Imp33.peg & Img29.jpeg & 1 & Negative ... \\
\hline 51 & Imp33.ppeg & Img3.jpeg & 1 & Negative ... \\
\hline 55 & Imp33.ipeg & Img50.ipeg & 1 & Negative ... \\
\hline 42 & Imp33.jpeg & Img25.ipeg & 1 & Negative ... \\
\hline 34 & Imp33.ipeg & Img36.ipeg & 1 & Negative ... \\
\hline 14 & Imp33.ipeg & Img32.ipeg & 1 & Negative ... \\
\hline 7 & Img33.peg & Img54.ipeg & 1 & Negative ... \\
\hline 20 & Imp33.ppeg & Img34.ipeg & 1 & Negative ... \\
\hline 31 & Ims33.jpeg & Img55.jpeg & 1 & Negative ... \\
\hline 32 & Imp33.jpeg & Img9.ppeg & 0 & Negative ... \\
\hline 29 & Imp33.jpeg & Img44.jpeg & 0 & Negative ... \\
\hline 30 & Imz33.jpeg & Img20.jpeg & 0 & Negative ... \\
\hline 25 & Imp33.ipeg & Img8.ppeg & 0 & Negative ... \\
\hline 26 & Imp33.peg & Img27.jpg & 0 & Negative ... \\
\hline 27 & Ims33.peg & Img31.jpeg & 0 & Negative ... \\
\hline 28 & Imp33.ppeg & Img13.ipeg & 0 & Negative ... \\
\hline 19 & Imp33.peg & Img58.jpg & 0 & Negative ... \\
\hline 17 & Ims33.jpeg & Img11.jpeg & 0 & Negative ... \\
\hline 18 & Imp33.peg & Img16.ipeg & 0 & Negative ... \\
\hline 21 & Imp33.ppeg & Img17.jpeg & 0 & Negative ... \\
\hline 22 & Img33.ipeg & Img48.ipeg & 0 & Negative ... \\
\hline
\end{tabular}

Fig 3.output image

In fig 4.the image keyword relationship chart represents the comparison of total image versus image keyword. It can be plotted after the bag annotation process. Here it can display the total number of relevant and irrelevant images to the user. In this fig apple keyword contains 13 relevant images. In keyword graph contains 2 relevant and 1 irrelevant images.

\section{Image Keyword Relationship}

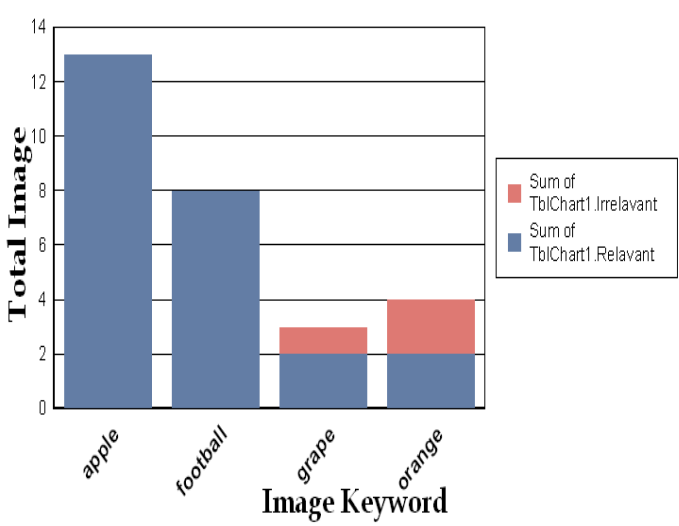

Fig.4.Keyword Relationship Chart
Fig 5 shows the relevant positive bag images. If the matching of source and match image percentage increases that could be stored in the positive bag and it is displayed to the user. The negative bag contains only the lower percentage of irrelevant images and it cannot be considered.

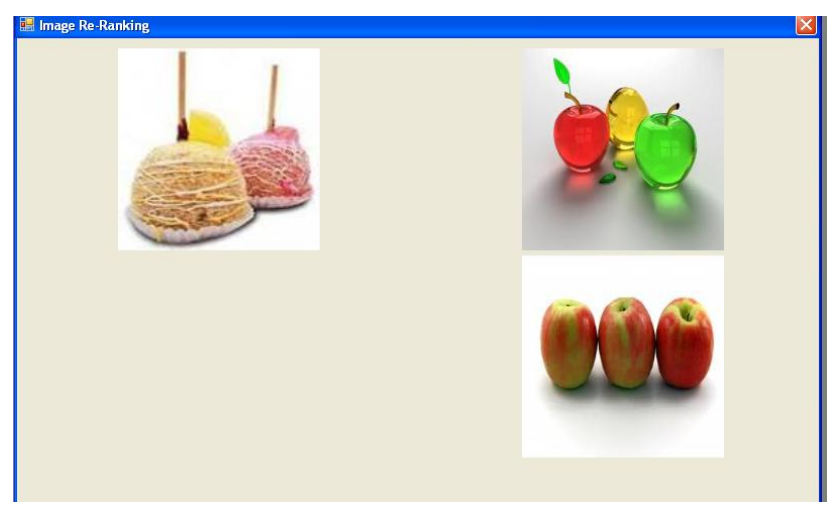

Fig.6.positive bag images

In fig. 6 the positive image ranking score can be displayed for every keyword. It shows the ranking percentage of comparison result with other images. Here every images ranking percentage can be varied according to the relevant images in the positive bag. The higher percentage of ranking score has the more relevant images which moves to the positive bag whereas below 70 percentage moves to the negative bag which contain irrelevant images. This provides an more efficient re-ranking of images compare to the existing method.

\section{Relavant Image Ranking Score}
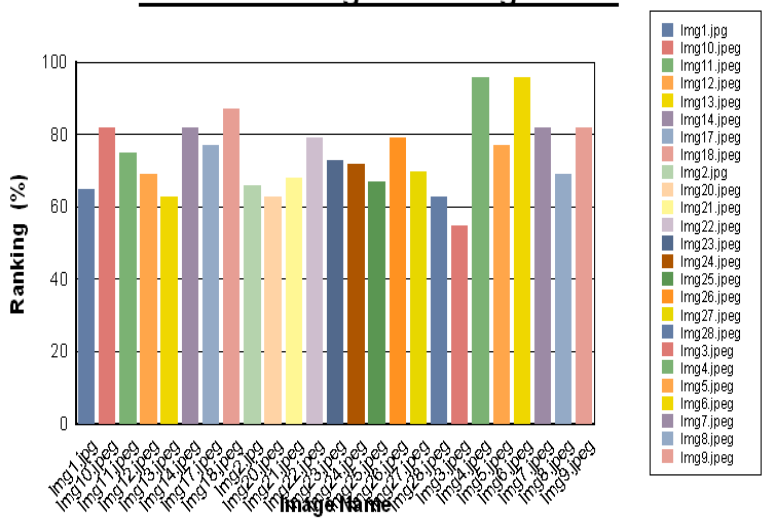

Fig.6.Image Ranking Score Chart

\section{CONCLUSION}

In this paper, we proposed a bag based frame work for large scale TBIR. By given a textual query, relevant images are to be re ranked after the initial text based search. To address the ambiguities in on the instances of both positive and negative bags, we develop Generalized Multiple Instance (GMI) to further enhance retrieval performance. Our framework using the automatic bag annotation method can achieve the best performance compared to other traditional image re-ranking methods. It also has the user log in for individual users which provide time consuming process for effective image retrieval. 


\section{REFERENCES}

[1] Xiao gang Wang, Ken Liu and Xiao Tang 'QuerySpecific Visual Semantic Spaces for Web Image Reranking'. In Proceeding of the 14th ACM International Conference on Multimedia,(2011).

[2] J. Cui, F. Wen,, and X. Tang: Real time Google and live image search re-ranking. In ProcACM Multimedia, (2008)

[3] jen-hao Hsiao., chu-song chen., and ming-syanchen : A novel language model based approach for image object mining and re-ranking. 2008 IEEE DOI 10.1109/ICDM (2008)

[4] Y. Rui, T. S. Huang, and S. Mehrotra: Content-based image retrieval withrelevance feedback in mars. In Proceedings of the IEEE InternationalConference on Image Processing, pages 815-818, 1997.

[5] W. H. Hsu, L. S. Kennedy., and S.-F. Chang:Video search reranking via information bottleneck principle. In Proceeding of the 14th ACMInternational Conference on Multimedia, pages 35-44, (2006).

[6] Z.-H. Zhou and H.-B. Dai:Exploiting image contents in web search. In Proceedings of the 20th International
Joint Conference on ArtificalIntelligence, pages 2928 2933, (2007).

[7] Y. Jing and S. Baluja: Textual query of personal photos facilitated by large scale web data. In Proceeding of the 17th International Conference on World Wide Web, pages 307-316,(2008).

[8] Florian Schroff., Antonio Criminisi., and Andrew Zisserman: Harvesting Image Databases from the Web. Proc. IEEE Conf.Computer Vision and Pattern Recognition, (2011).

[9] MattiaBroilo:A Stochastic Approach to Image Retrieval Using Relevance Feedback and Particle Swarm OptimizationIn Proceedings 10th Workshop on Multimedia Signal Processing-MMSP, Cairns, Australia, (2008)

[10] R. Yan., A. G. Hauptmann., and R. Jin: Multimedia search withpseudo-relevance feedback. In Proceedings of the ACM International Conference on Image and Video Retrieval, pages 238-247, (2003).

[11] Y. Jing and S. Baluja: Pagerank for product image search. In Proceeding of the 17th International Conference on World Wide Web, pages 307-316, (2008). 